\title{
Extracción atraumática de implantes: predictibilidad de la técnica y del implante reimplantado
}

\author{
Eduardo Anitua*, MD, DDS, $\mathrm{PhD}_{1}$
}

${ }_{1}$ Eduardo Anitua Foundation, Vitoria, Álava, España

Recibido: 22 de enero del 2016 Aprobado: 29 de febrero del 2016

*Autor de correspondencia: Eduardo Anitua. Dirección: C/ José María Cagigal 19, 10005 Vitoria, Álava, España. Teléfono: +34 945160650. Correo electrónico: lapica77@gmail.com

Cómo citar este artículo: Anitua E. Extracción atraumática de implantes: predictibilidad de la técnica y del implante reimplantado. Rev Nac Odontol. 2016;12(23):23-30. doi: 10.16925/od.v12i23.1407

Resumen. Introducción: la reversibilidad del tratamiento implantológico es, hoy en día, uno de los puntos clave para el retratamiento de casos en los que se han producido peri-implantitis o pérdidas óseas que generan un fracaso de los implantes. Un sistema que permita la extracción de los implantes de forma atraumática y la nueva inserción de implantes en una misma fase quirúrgica resuelve el problema de forma eficaz. Método: se realizó un estudio piloto sobre nueve pacientes en los que hicieron explantaciones e inserción de implantes en la misma fase quirúrgica y localización. Estos implantes reinsertados fueron seguidos en el tiempo para evaluar su supervivencia. Resultados: los implantes se siguieron durante 50+/-2 meses desde su inserción (rango de 48-52 meses) y durante 43+/- 3 meses desde la carga (rango de 40-48 meses). Durante el seguimiento, no se registró ningún implante fracasado. La pérdida ósea mesial fue de 1,0+/-0,8 mm y la distal fue de 1,0+/-0,8 $\mathrm{mm}$. Conclusiones: la técnica de explantación atraumática es una técnica segura y predecible que puede ser utilizada sin riesgos en la mayor parte de los implantes comercializados.

Palabras clave: explantación, implante, peri-implantitis, reimplantado. 


\title{
Atraumatic Extraction of Implants: Predictability of the Technique and of the Re-Implanted Implant
}

\begin{abstract}
Introduction: The reversibility of the implant treatment is, currently, one of the key points for successive treatment of cases where peri-implantitis or bone loss occur that produce a failure of the implants. A system that allows the extraction of the implants in an atraumatic manner and the insertion of new implants in the same surgical phase resolves the problem in an efficient manner. Method: A pilot study was performed on nine patients where explantation and implant grafting were done in the same surgical and localization phase. These reinserted implants were followed up over time to evaluate its survival. Results: The implants were followed up during $50+/-2$ months from the time of its insertion (range of 48-52 months) and during 43+/-3 months from the charge (range of 40-48 months). No implant failure was registered during the follow up time. The mesial bone loss was of $1,0+/-0,8 \mathrm{~mm}$ and the distal bone was of $1,0+/-0,8 \mathrm{~mm}$. Conclusion: The technique of atraumatic explantation is a safe and predictive technique that may be used without risks in most commercial implants.
\end{abstract}

Keywords: explantation, implants, peri-implantitis, reimplants.

\section{Extração de implantes atraumática: previsibilidade da técnica e do implante reimplantado}

Resumo. Introdução: Atualmente, a reversibilidade do tratamento implantológico é um dos pontos chave para o retratamento de casos nos quais se tem produzido periimplantite ou perdas ósseas que geram um falimento dos implantes. Um sistema que permita a extração dos implantes de forma atraumática e a nova inserção de implantes em uma mesma fase cirúrgica resolve eficazmente o problema. Método: realizou-se um estudo piloto em nove pacientes nos quais fizeram explantações e inserção de implantes na mesma fase cirúrgica e localização. Esses implantes reinseridos foram seguidos no tempo para avaliar sua supervivência. Resultados: os implantes seguiram durante 50+/-2 meses desde sua inserção (faixa de 48-52 meses) e durante 43+/- 3 meses desde a carga (faixa de 40-48 meses). Durante o acompanhamento, não se registrou nenhum implante falido. A perda óssea mesial foi de $1,0+/-0,8 \mathrm{~mm}$ e a distal foi de 1,0+/-0,8 mm. Conclusões: a técnica de explantação atraumática é uma técnica segura e previsível que pode ser utilizada sem riscos na maioria dos implantes comercializados.

Palavras chave: explantação, implante, periimplantite, reimplantado. 


\section{Introducción}

La explantación de implantes fracasados por distintos motivos es un reto creciente en nuestras consultas. Para dar solución a este nuevo ítem, se han elaborado diferentes técnicas, al margen de las técnicas de trefinado completo del implante y de retirada del implante con un cilindro óseo alrededor, consideradas como técnicas clásicas.

En la literatura internacional, encontramos trabajos de investigación que reportan técnicas de extracción de implantes. Los trabajos de Covani y colaboradores [1, 2], desarrollados en los años 2006 y 2009 , muestran una técnica más conservadora que las trefinas convencionales para remover implantes con una fresa a bajas revoluciones bajo irrigación capaz de retirar el hueso alrededor del implante. Al retirar el hueso se rompe la unión estructural de la integración y puede retirarse el implante. Esta técnica, a pesar de ser más conservadora que la convencional por trefinado, deja defectos de mayor diámetro que el del implante extraído, por lo que se pierde parte del lecho óseo.

Técnicas basadas en el contratorque han sido empleadas en humanos en la literatura para desinsertar implantes de diámetro pequeño, como lo reportado por Simon y Caputo [3]. Estos dos autores utilizan un dispositivo para retirar los implantes sin la pieza extractora intermedia. A pesar de ser implantes de diámetro reducido, se encontraron varios efectos negativos como: fractura, deformación del implante y fractura de fragmentos óseos.

La técnica reportada por nuestro grupo de estudio [4] permite la desinserción atraumática a contratorque del implante, dejando un lecho óseo totalmente conservado. Los extractores desinsertan el implante sin dañar la zona en la que estaba y posibilitan, en muchas ocasiones, la inserción de un nuevo implante en el mismo lugar y acto quirúrgico.

Además, el seguimiento de estos implantes insertados en la zona de la explantación previa no está bien documentado. Son muchos los estudios que reportan casos de implantes inmediatos posextracción dental, pero los estudios que describan el seguimiento de los implantes insertados en el mismo sitio de una explantación dental en el mismo acto quirúrgico son escasos $[5,6]$.

\section{Materiales y métodos}

Se realizaron explantaciones con la utilización del kit de explantación (BTi Biotechnology Institute, Vitoria, España). El kit consta de una llave que se utiliza para transmitir la fuerza de contratorque al implante por medio de una pieza conectora (extractor) que se inserta en la conexión del implante. La llave está tarada a $200 \mathrm{ncm}$ para que cuando llegue a este par de fuerza se desarme automáticamente, con lo cual se evita generar lesiones por cizallamiento o roturas óseas derivadas de pares de fuerza superiores a los $200 \mathrm{ncm}$, o por fractura del extractor en el interior del implante. Cuando la llave se desarma, puede rearmarse mediante una fuerza de palanca contraria al acodamiento en un cilindro plástico que permite su introducción y enderezamiento [7] (figura 1).

El extractor es posicionado mediante una llave de encastre manual en sentido antihorario y luego con la llave se ejerce una fuerza antihoraria (contratorque) sobre el conjunto implante-extractor, que generará la ruptura de la unión hueso-implante, produciéndose la desoseointegración (figuras 2 y 3 ).

En casos en los que se alcanzan más de 200 ncm (el torque de desinserción), que permite la llave y que no sea posible continuar con la explantación, se realiza un trefinado ultrafino en torno al implante (1-2 mm) del hueso más coronal. Después se vuelve a intentar la maniobra con un nuevo extractor. La eliminación de estos primeros milímetros de hueso cortical hace que el torque de desinserción caiga drásticamente y que, por ello, ahora seamos capaces de extraer el implante con un contratorque inferior a los $200 \mathrm{ncm}$ [7] (figuras 4 y 5).

Una vez insertados los nuevos implantes, se les hizo un seguimiento para evaluar la predictibilidad del implante reimplantado. 


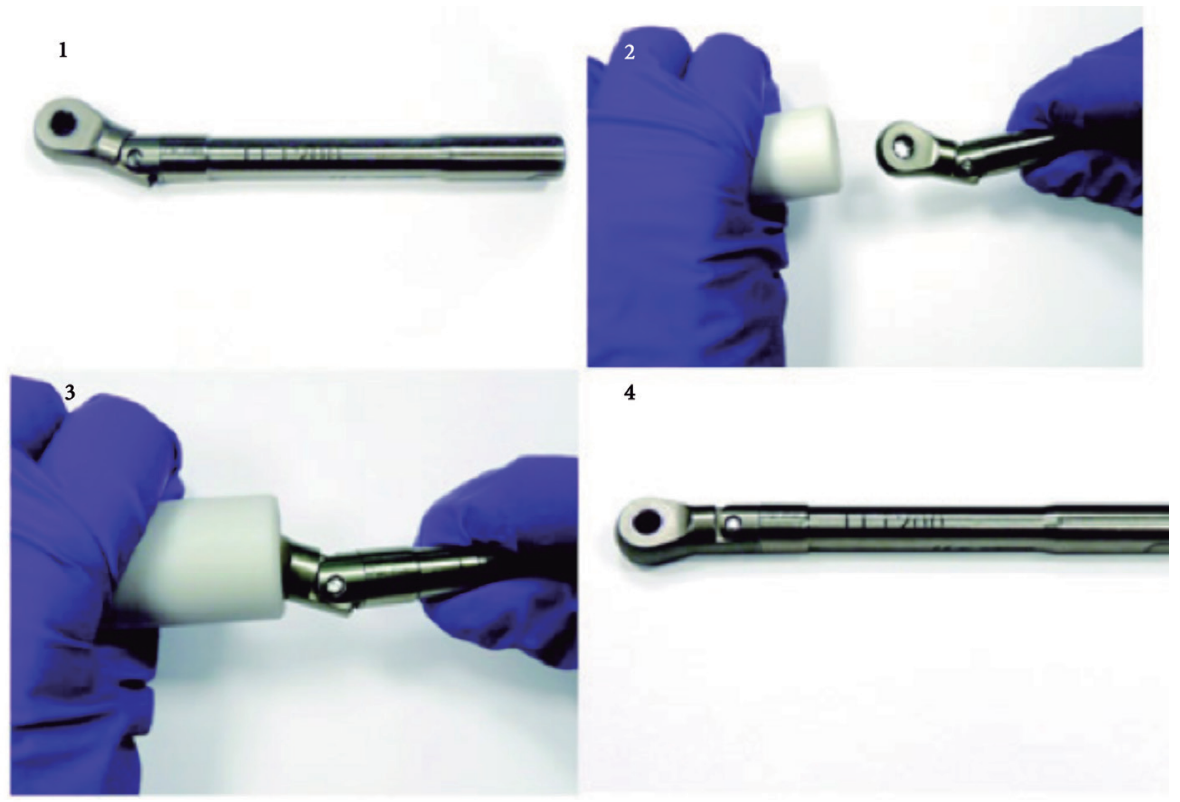

Figura 1. 1) Llave que se ha desactivado al llegar a los $200 \mathrm{ncm}$. 2) Para revertir la desactivación y poder utilizarla de nuevo, la introducimos en el tubo plástico de rearme. 3) Hacemos presión para llevar la llave a su posición original (armada). 4) Extraemos la llave del tubo plástico Fuente: elaboración propia
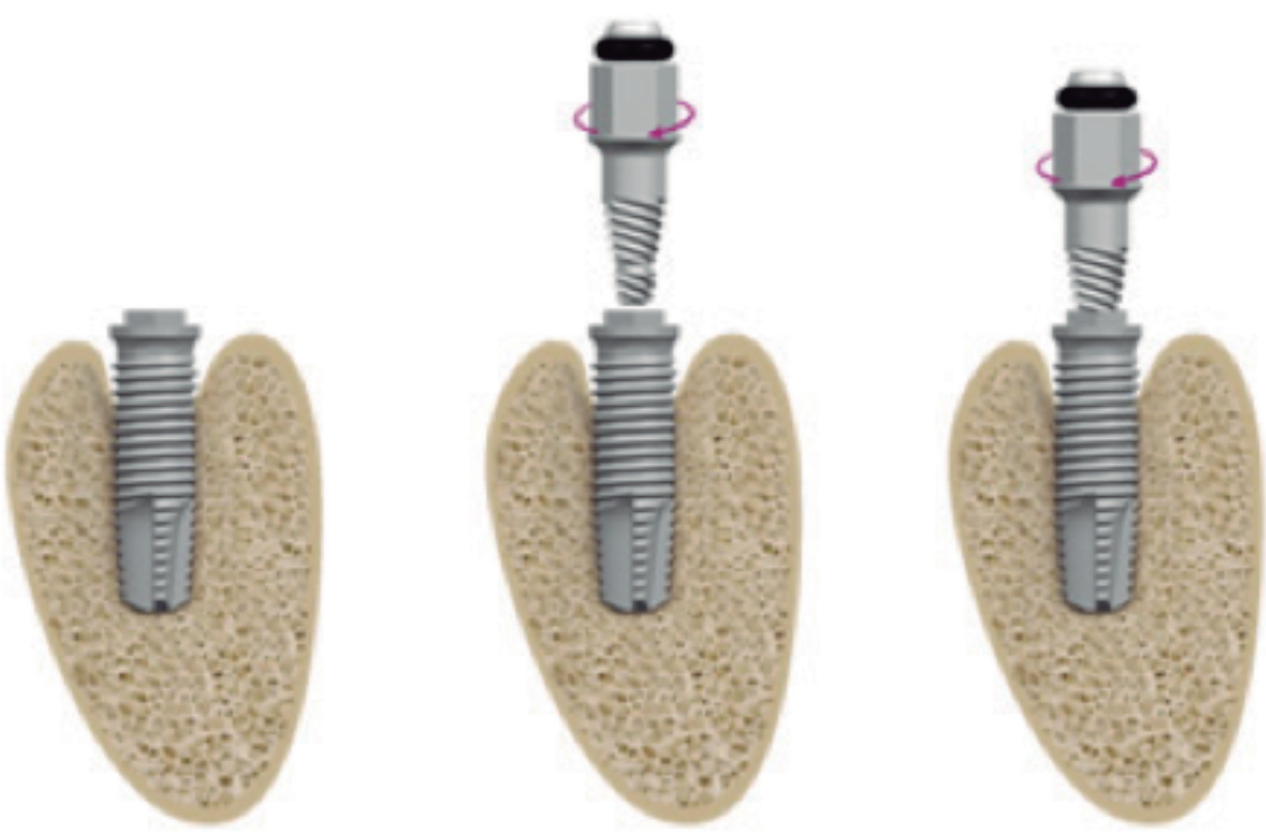

Figura 2. Introducción del extractor en sentido antihorario en la conexión del implante Fuente: elaboración propia 


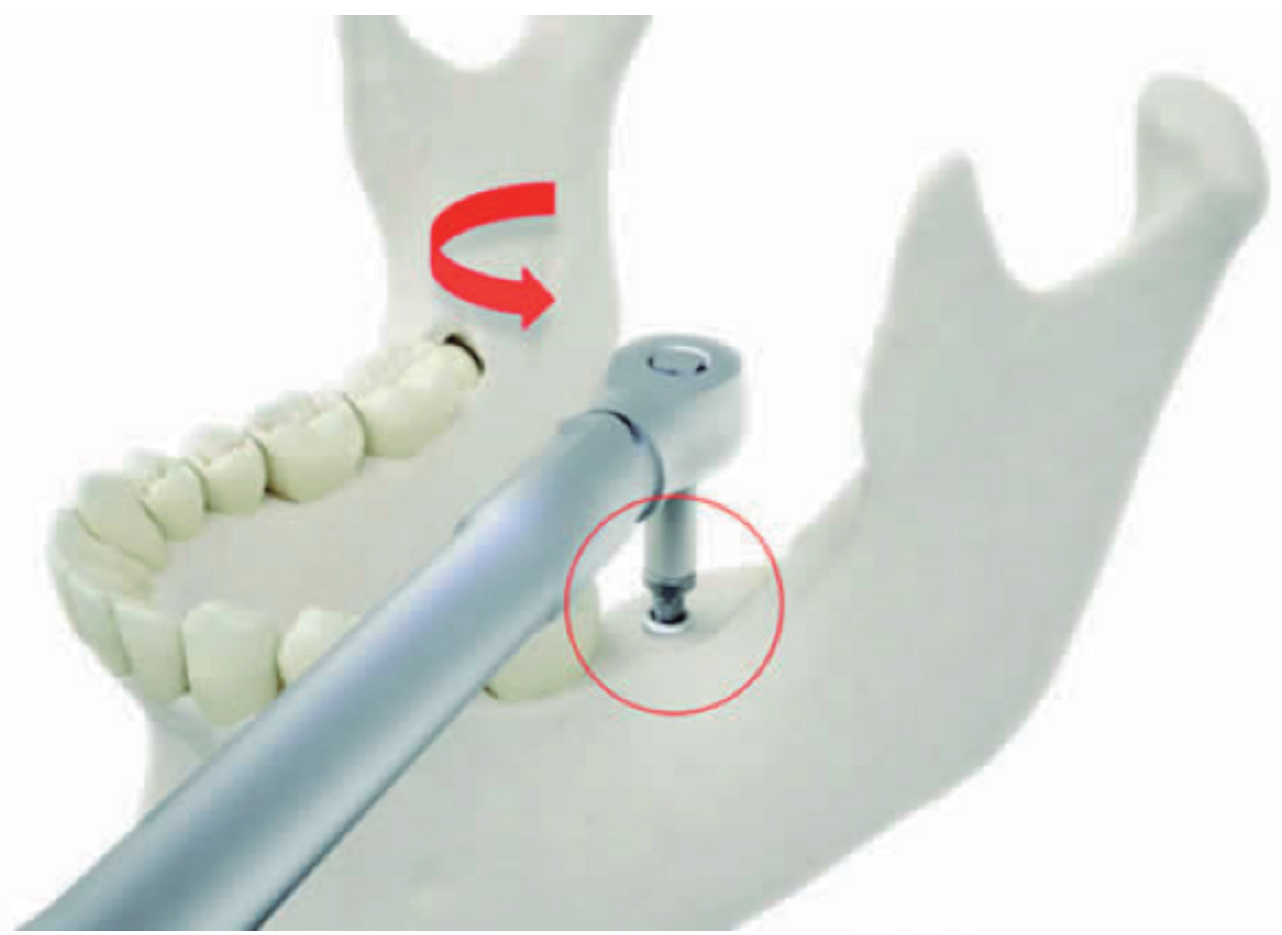

Figura 3. Continuamos el movimiento antihorario con la llave de torque. Es importante que este movimiento sea axial al implante en todo momento

Fuente: elaboración propia

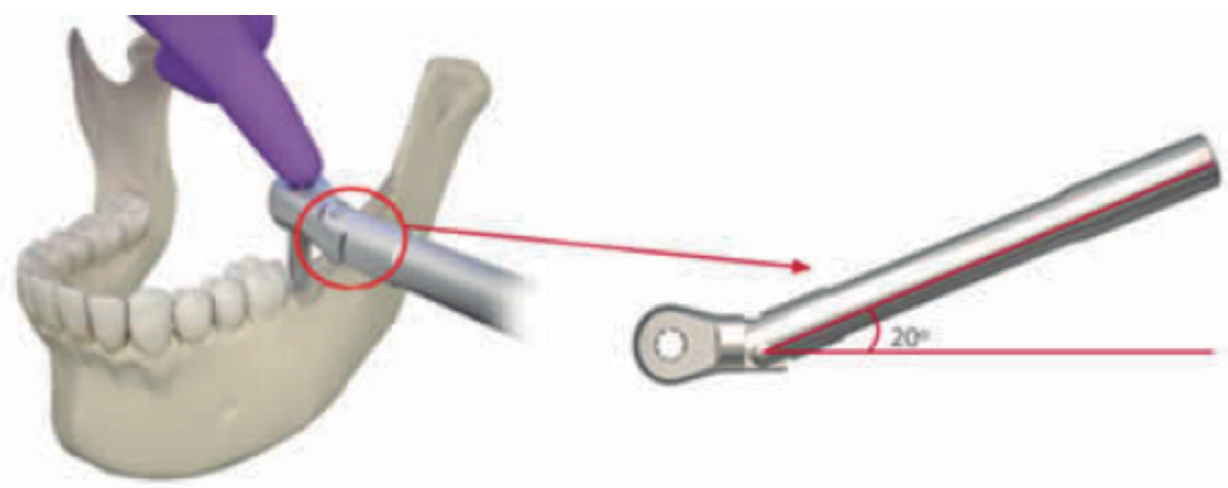

Figura 4. Desarticulación de la llave de torque que nos indica que hemos llegado a los $200 \mathrm{ncm}$ Fuente: elaboración propia 

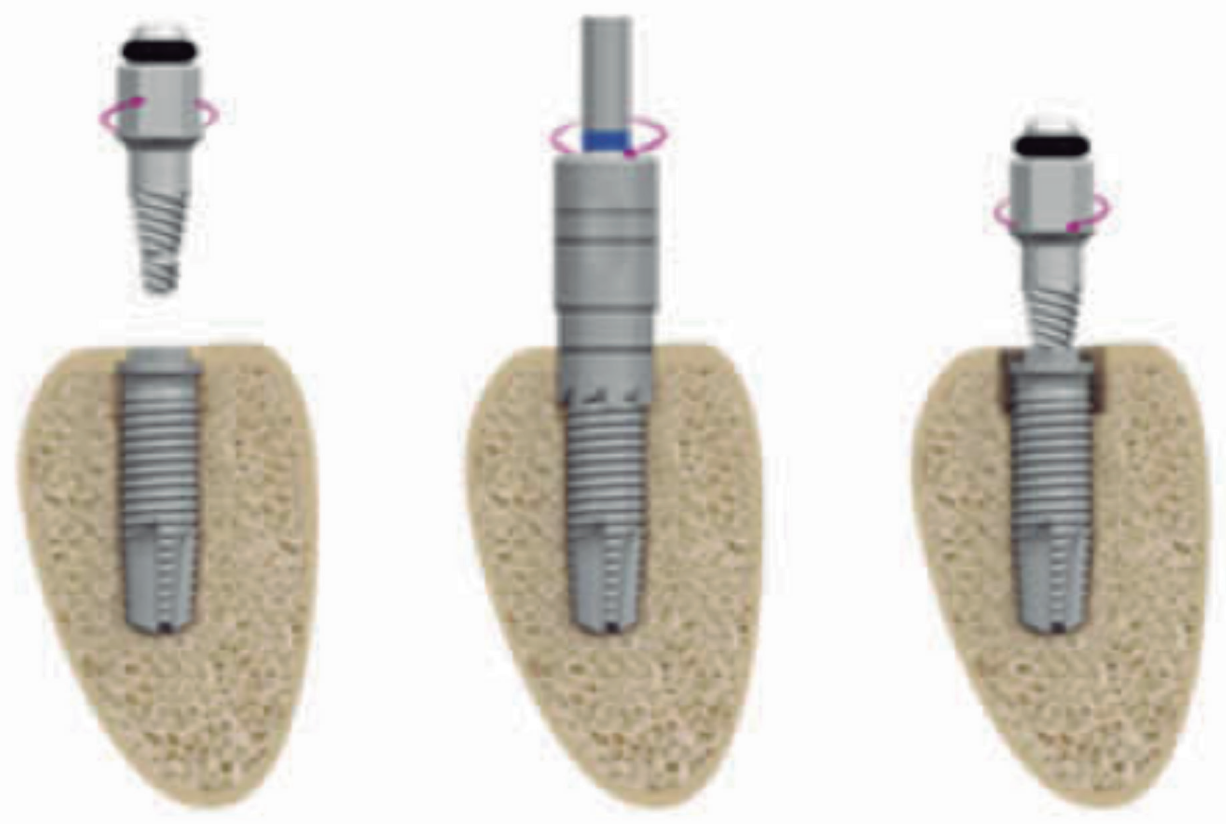

Figura 5. Trefinado posterior de los 2 o 3 primeros milímetros del implante para romper la unión cortical de las primeras espiras, y posterior intento de desinserción del implante con el extractor Fuente: elaboración propia

\section{Resultados}

Se hicieron nueve explantaciones en nueve pacientes a quienes se les insertaron nueve implantes en el mismo lecho y acto quirúrgico. Seis de los pacientes fueron mujeres, con una media de edad de $61 \pm 4$ años. Seis de los implantes se insertaron en el maxilar superior y tres en la mandíbula. La media del torque de extracción de los implantes fracasados fue de $162 \pm 41 \mathrm{ncm}$. Los implantes insertados en los lechos posexplantación presentaron un torque medio de $36 \pm 16 \mathrm{ncm}$. Únicamente dos implantes fueron insertados con un torque inferior a $15 \mathrm{ncm}$.

Dos de los implantes fueron cortos $(5,5 \mathrm{~mm}$ x 5,5 mm y 5,5 mm x 7,5 mm). Tres de los implantes fueron de $8,5 \mathrm{~mm}$ de longitud con diámetros de 4, 4,5 y 5,5 $\mathrm{mm}$. El resto de los implantes fueron de 10 a $13 \mathrm{~mm}$ de longitud y de 3,75 a $5 \mathrm{~mm}$ de diámetro. Los implantes se siguieron durante 50 \pm 2 meses desde su inserción (rango 48-52 meses) y de $43 \pm 3$ meses desde la carga (rango de 40 a 48 meses). Durante el seguimiento, no se registró ningún implante fracasado. La pérdida ósea mesial fue de $1,0 \pm 0,8 \mathrm{~mm}$ y la distal de $1 \pm 0,8 \mathrm{~mm}$.

\section{Discusión}

Son muchos los estudios que reportan casos de implantes inmediatos posextracción dental, pero los estudios que describan el seguimiento de los implantes insertados en el mismo sitio de una explantación dental en el mismo acto quirúrgico son escasos.

Los primeros aportes sobre la posibilidad de reimplantación dental tras una explantación en humanos y el seguimiento del implante reimplantado corren a cargo de Covani y colaboradores $(2006,2009$ y 2010) [1, 2, 5]. En estos estudios, se hacen explantaciones conservando al máximo el hueso alveolar del lecho peri-implantar por medio de la separación del hueso en contacto con el implante mediante fresado con una fresa fina a baja velocidad con irrigación. Posteriormente, se insertaban los implantes siguiendo las especificaciones técnicas de los fabricantes con un nuevo fresado para preparar un nuevo lecho ligeramente más ancho a fin de estabilizar el implante. Todos los implantes tratados mediante este método fueron implantes cilíndricos y roscados que se retiraron por fractura a diferentes niveles. 
El número total de casos recogidos es de nueve implantes en nueve pacientes. Todos ellos fueron rehabilitados tras el periodo de oseointegración, sin mostrar en la re-entrada ningún signo de fracaso temprano. Después de su rehabilitación protésica, se hizo un seguimiento de seis meses de todos los implantes (12 meses en total desde su inserción), tras lo cual no hubo fracaso de ninguno de los implantes ni pérdidas óseas mayores a las descritas como normales tras la carga.

La segunda referencia bibliográfica que encontramos en la literatura es la reportada por Grossmann y Levin en el 2007 [6]. En este estudio, se hizo el seguimiento de 31 implantes reimplantados en el lugar de explantación de un implante anterior, insertados en 28 pacientes. El tiempo medio de seguimiento fue de 19,4 meses desde la inserción con un rango situado entre los seis y los 46 meses. Durante el periodo de seguimiento, nueve de los implantes reimplantados fracasaron, por lo que su tasa de supervivencia se situó en el 71\%, inferior a las tasas de supervivencia de los implantes insertados de forma convencional. Todos los fracasos sucedieron en el primer año tras la inserción.

En nuestro estudio, ninguno de los implantes insertados en zonas con peri-implantitis previa ha fracasado. Así, la inserción inmediata de un nuevo implante para el tratamiento de implantes fracasados podría ser una alternativa que disminuye costes, tiempo y morbilidad quirúrgica. La técnica de explantación atraumática es una técnica segura y predecible que puede ser utilizada sin riesgos en la mayor parte de los implantes comercializados.

En el siguiente caso clínico, mostramos una paciente del estudio a la que se le hicieron explantaciones e implantes en las zonas de las extracciones de implantes (figuras 6-10).
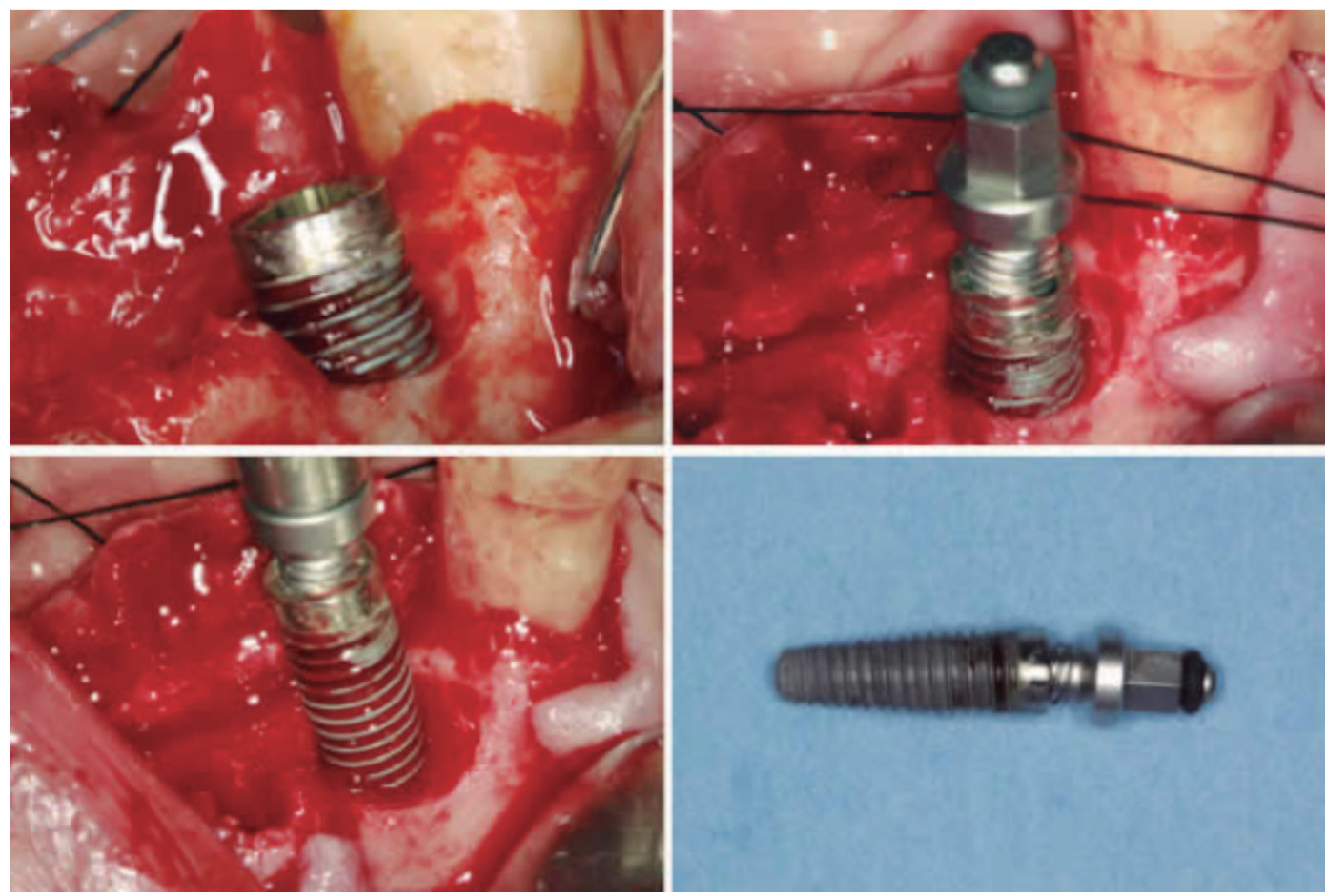

Figura 6. Imágenes iniciales del caso en las que puede observarse la situación del implante en posición 34 Fuente: elaboración propia 


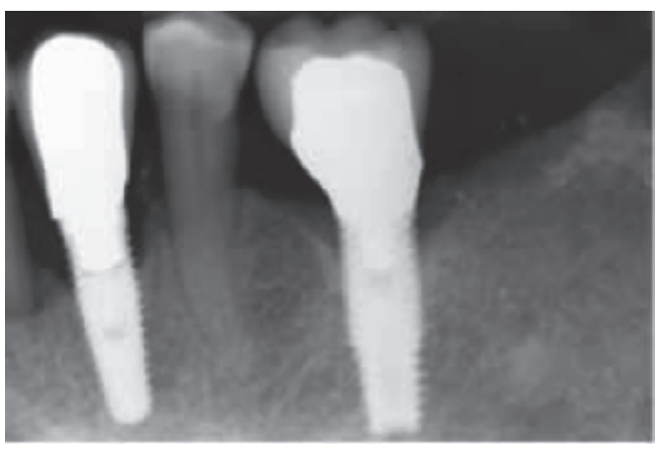

Figura 7. Radiografía inicial. Podemos observar la mala situación periodontal del frente antero-inferior. Se decide, además de la explantación del implante en posición 34, la extracción de los dientes afectados y la inserción de implantes en la zona Fuente: elaboración propia

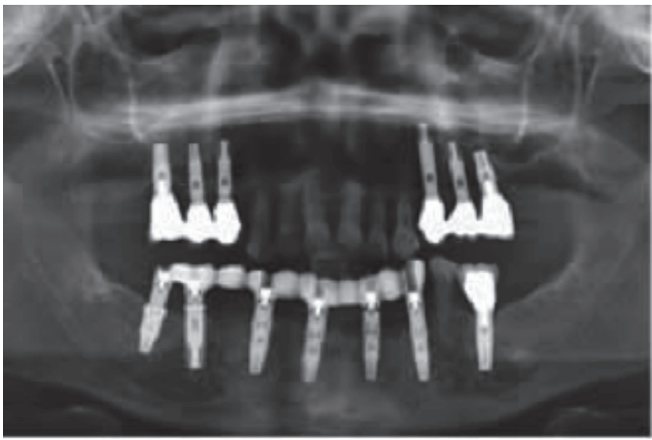

Figura 9. Imagen radiográfica con la prótesis definitiva al año. Podemos ver la estabilidad de todos los implantes insertados Fuente: elaboración propia

\section{Conclusiones}

La técnica de explantación atraumática es una técnica segura y predecible que puede ser utilizada sin riesgos en la mayor parte de los implantes comercializados. La inserción de los implantes en el mismo acto y lecho quirúrgico debe ser tomada en consideración y analizada junto con otros factores dependientes del lecho receptor y del paciente, a fin de conseguir tasas de éxito similares a las de los implantes insertados en un lecho primario sin tratamientos previos.

\section{Referencias}

[1] Covani U, Barone A, Cornelini R, Crespi R. Clinical Outcome of implants placed immediately after implant removal. J Periodontol. 2006;4:722-7.

[2] Covani U, Marconcini S, Crespi R, Barone A. Immediate implant placement after removal of failed

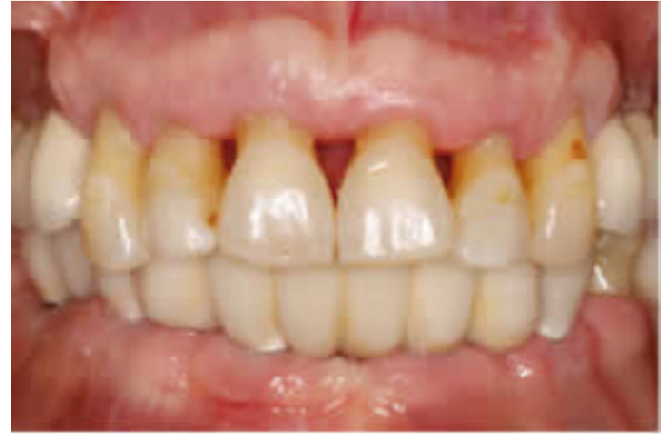

Figura 8. Imagen de la paciente con la prótesis definitiva.

Podemos observar el estado de los tejidos peri-implantarios y periodontales

Fuente: elaboración propia

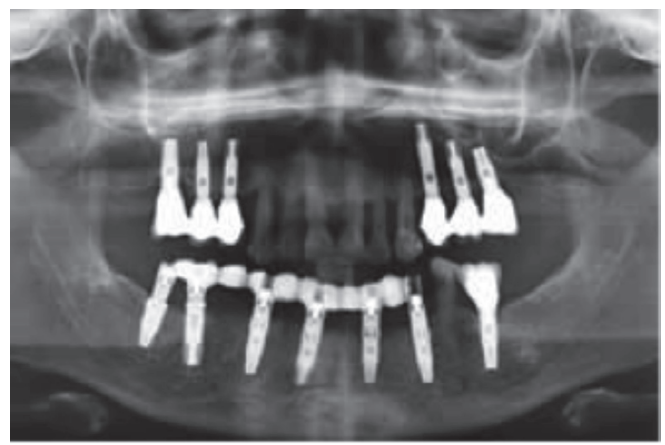

Figura 10. Imagen radiográfica a los tres años. La estabilidad de los tejidos se mantiene

Fuente: elaboración propia

implant: a clinical and histological case report. J Oral Implantol. 2009;4:189-95.

[3] Simon H, Caputo AA. Removal torque of immediately loaded transitional endosseous implants in human subjects. Int J Oral Maxillofac Implants. 2002;17:839-45.

[4] Anitua E, Orive G. A new approach for atraumatic implant explantation and immediate implant installation. Oral Surg Oral Med Oral Pathol Oral Radiol 2012;113:e19-e25.

[5] Covani U, Marconcini S, Santini S, Cornelini R, Barone A. Immediate restoration of single implants placed immediately after implant removal. A casereport. Int J Periodontics Restorative Dent. 2010 Dec;30(6):639-45.

[6] Grossmann Y, Levin L. Sucess and survival of single dental implants placed in sites of previously failed implants. J Periodontol. 2007;78:1670-4.

[7] Anitua E, Piñas L, Begoña L, Alkhraisat MH. Prognosis of dental implants immediately placed in sockets affected by periimplantitis: A retrospective pilot study. Int J Periodontics Restorative DENT. 2015 [IN PRESS]. 